\title{
Adsorption Behavior of Plasma Proteins on Polyaminoacid Membrane Surface
}

\author{
Dedicated to the Memory of the late Professor Ichiro Sakurada
}

\author{
Akio NAKAJIMA and Yoshio HATA* \\ Department of Applied Chemistry, Osaka Institute of Technology, \\ Ohmiya, Asahi-ku, Osaka 535, Japan \\ * Research Center for Medical Polymers and Biomaterials, \\ Kyoto University, Kyoto 606, Japan
}

(Received January 15, 1987)

\begin{abstract}
A kinetic equation describing two consecutive adsorption steps was derived by considering the diffusion-controlled step followed by the energy barrier-controlled step. A novel method to evaluate the interfacial pressure of protein solution on polymer membrane surface as a function of time was developed. The adsorption behavior of bovine serum albumin and bovine serum $\gamma$-globulin onto block and random polyaminoacid copolymer membrane surface was investigated by using the equation and experimental method developed. Comparison of adsorption behavior was made on block and random copolymers composed of $\gamma$-benzyl-L-glutamate and Lleucine, and partly hydrophilic polyaminoacid of different $\mathrm{OH}$ contents which were prepared by aminoalcoholysis of the parent block copolymer. The effective cross-sectional area $A$ of albumin $\left(c a .600 \mathrm{~A}^{2}\right)$ was about three times of $\gamma$-globulin $\left(c a .200 \mathrm{~A}^{2}\right)$ for both block and random copolyaminoacids. The $\mathrm{F}_{\mathrm{ab}}$ portion of $\gamma$-globulin seemed to be oriented toward hydrophilic surface, in contrast to the orientation of $F_{c}$ portion toward hydrophobic surface.
\end{abstract}

KEY WORDS Adsorption / Surface Pressure / Surface Area / Contact Angle / Interfacial Tension / Albumin / Globumin / Polyaminoacids /

Upon introduction of a synthetic polymer material into cardiovascular system, rapid adsorption of plasma proteins takes place as an initial event in a complex series of reactions. The purpose of this research is to analyse the time-dependent protein adsorption from solution on polyaminoacid membrane surface from purely physico-chemical aspects, and to discuss effects of surface structure on the adsorption behavior.

The driving force for protein adsorption decreases with decreasing interfacial free energy. Thus the protein adsorption process will be kinetically pursued from time-dependency of the solid/liquid interfacial pressure. The adsorption process leading to the equilibrium may be treated by assuming two ratedetermining steps. The first step is the diffusion-controlled adsorption of protein molecules onto the surface from bulk solution at the very beginning of the process. As adsorption proceeds, the interfacial tension is reduced and thus the interfacial pressure is raised. So that, in the second step, the adsorption rate is controlled by the energy barrier to be overcome.

In the kinetic treatments ${ }^{1-7}$ reported so far, these two steps were separately formulated, and no paper did clarify the transition between these two rate-determining processes. In this paper, first we discuss the adsorption kinetics taking into account the transpose of these two 
steps, and secondly we analyse the adsorption behavior of plasma proteins to the polymer solid/liquid interface by the use of equation proposed by us and a novel experimental method to obtain the surface pressure and the surface area per protein molecule.

\section{ADSORPTION KINETICS}

As is illustrated in Figure 1, we assume three phases, i.e., interface, subphase, and bulk phase. Now we take $\mathrm{x}$-axis vertically to the surface and indicate the protein concentrations in subphase and bulk phase respectively by $c$ $(0, t)$ and $c(x, t)$, where $t$ denotes the time. The initial concentration $c_{\mathrm{o}}$ of protein is equal to $c(x, 0)$, and the number of protein molecules adsorbed per unit area is indicated by $\Gamma(t)$. With such a model, the diffusion-controlled process and energy barrier-controlled process are respectively operated between bulk phase and subphase, and between subphase and interface.

Regarding the diffusion-controlled process, by solving ${ }^{8-10}$ Fick's second law with initial conditions, $c(x, 0)=c_{\mathrm{o}}$ and $\Gamma(0)=0$, and boundary condition $c(\infty, t)=c_{\mathrm{o}}$, together with $\mathrm{d} \Gamma / \mathrm{d} t=D(\partial c / \partial x)_{x=0}$, we obtain the concentration in subphase as:

$$
c(0, t)=c_{\mathrm{o}}-\frac{1}{\sqrt{\pi D}} \int_{0}^{t} \frac{\Gamma^{\prime}(\tau)}{\sqrt{t-\tau}} \mathrm{d} \tau
$$

where $\Gamma^{\prime}=\mathrm{d} \Gamma / \mathrm{d} t, D$ is the diffusion constant, and $\pi=3.142$.

Ward and Tordai, ${ }^{2}$ taking into account the energy barrier-controlled process together with desorption process for two phase system composed of bulk phase and interface, arrived at the following equation:

$$
\frac{\mathrm{d} \Gamma}{\mathrm{d} t}=k_{1} c_{\mathrm{o}} \exp \left(-\frac{\Pi A}{k T}\right)-k_{2} \Gamma
$$

where $k_{1}$ and $k_{2}$ are the rate constants for adsorption and desorption, respectively, and $k$ is the Boltzman constant. The energy barrier to be crossed over to creat a space of area $A$ in

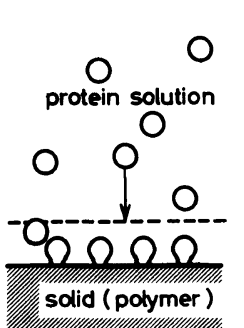

(a)

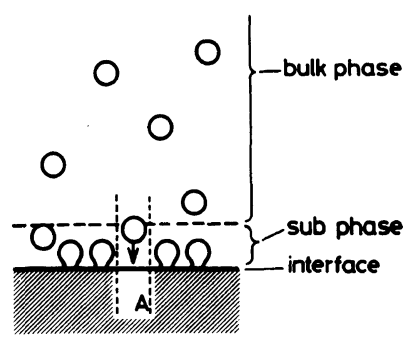

(b)
Figure 1. Schematic representation for protein adsorption on polymer film surface.

a surface film of surface pressure $\Pi$ in order to adsorb a molecule or segment would be $\Pi A$. Ward and Tordai concluded that the adsorption velocity is directly proportional to the bulk concentration $c_{\mathrm{o}}$.

According to our model shown in Figure 1, $\mathrm{d} \Gamma / \mathrm{d} t$ is proportional to the protein concentration $c(0, t)$ in subphase:

$$
\frac{\mathrm{d} \Gamma}{\mathrm{d} t}=k_{1} c(0, t) \exp \left(-\frac{\Pi A}{k T}\right)-k_{2} \Gamma
$$

Combining eq 1 , and 3 , and assuming $\mathrm{d} \Gamma / \mathrm{d} \Pi$ is independent of $t$, finally we obtain:

$$
\begin{aligned}
\frac{\mathrm{d} \Pi}{\mathrm{d} t}= & k_{1}\left\{c_{\mathrm{o}}\left(\frac{\mathrm{d} \Gamma}{\mathrm{d} \Pi}\right)^{-1}\right. \\
& \left.-\frac{1}{\sqrt{\pi D}} \int_{0}^{t} \frac{\Pi^{\prime}(\tau)}{\sqrt{t-\tau}} \mathrm{d} \tau\right\} \exp \left(-\frac{\Pi A}{k T}\right)-k_{2} \Pi
\end{aligned}
$$

where $\Pi^{\prime}$ indicates $\mathrm{d} \Pi / \mathrm{d} t$.

For multi-component protein system, eq 4 is extended to an equation system by assuming $\Pi=\Sigma \Pi_{\mathrm{i}}$.

Now we demonstrate some simulation results. For $t \cong 0, \Pi$ becomes nearly 0 , and the numerical solution of $\Pi$ is given by:

$$
\begin{aligned}
\Pi= & c_{\mathrm{o}}\left(\frac{\mathrm{d} \Gamma}{\mathrm{d} \Pi}\right)^{-1} \\
& \times \sqrt{D}\left\{2 \sqrt{\frac{t}{\pi}}-\frac{1}{\mu}\left(1-\mathrm{e}^{\mu^{2} t} \operatorname{erfc} \sqrt{\mu^{2} t}\right)\right\}
\end{aligned}
$$




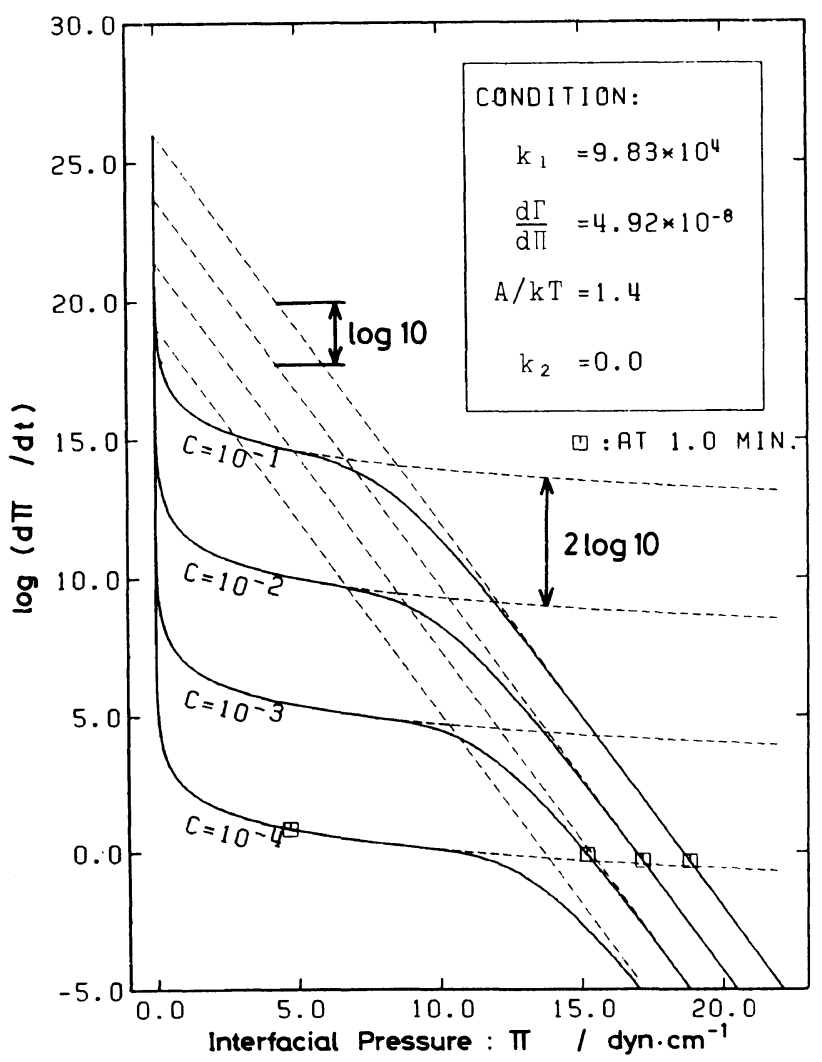

Figure 2. Dependence of calculated $\log (\mathrm{d} \Pi / \mathrm{d} t)$ on interfacial pressure $\Pi$ for various values of bulk concentration $c_{\mathbf{o}}$.

where $\mu=k_{1} / \sqrt{D}$. Further, the differential of $\Pi$ with $t$ is given by:

$$
\frac{\mathrm{d} \Pi}{\mathrm{d} t}=k_{1} c_{\mathrm{o}}\left(\frac{\mathrm{d} \Gamma}{\mathrm{d} \Pi}\right)^{-1} \mathrm{e}^{\mu^{2} t} \text { erfc } \sqrt{\mu^{2} t}
$$

If $k_{1} \gg D$, then equilibrium between interface and subphase is accomplished instantaneously, thus $c(0, t)$ becomes 0 , and eq 6 is approximated as:

$$
\frac{\mathrm{d} \Pi}{\mathrm{d} t}=c_{\mathrm{o}}\left(\frac{\mathrm{d} \Gamma}{\mathrm{d} \Pi}\right)^{-1} \sqrt{\frac{D}{\pi t}}
$$

which corresponds to the solution ${ }^{11}$ of widely used diffusion equation.

On the other hand, for $t \gg 0$, the second term of the right hand of eq 1 is neglected, and eq 4 is reduced to:

$$
\frac{\mathrm{d} \Pi}{\mathrm{d} t}=k_{1}\left(\frac{\mathrm{d} \Gamma}{\mathrm{d} \Pi}\right)^{-1} c_{\mathrm{o}} \exp \left(-\frac{\Pi A}{k T}\right)-k_{2} \Pi
$$

In Figure 2, for irreversible adsorption $\left(k_{2}=0\right)$, the numerical solution curves obtained by eq 4 for various $c_{\mathrm{o}}$ values are indicated by solid curves, and the results obtained by eq 6 (diffusion-controlled) and 8 (energy barrier-controlled) are shown by broken straight lines and broken curves, respectively. Thus we can determine $A$ from $\log (\mathrm{d} \Pi / \mathrm{d} t)$ vs. $\Pi$ curves.

\section{EXPERIMENTAL}

\section{Materials}

The protein samples used for adsorption were Bovine serum albumin BSA and Bovine 


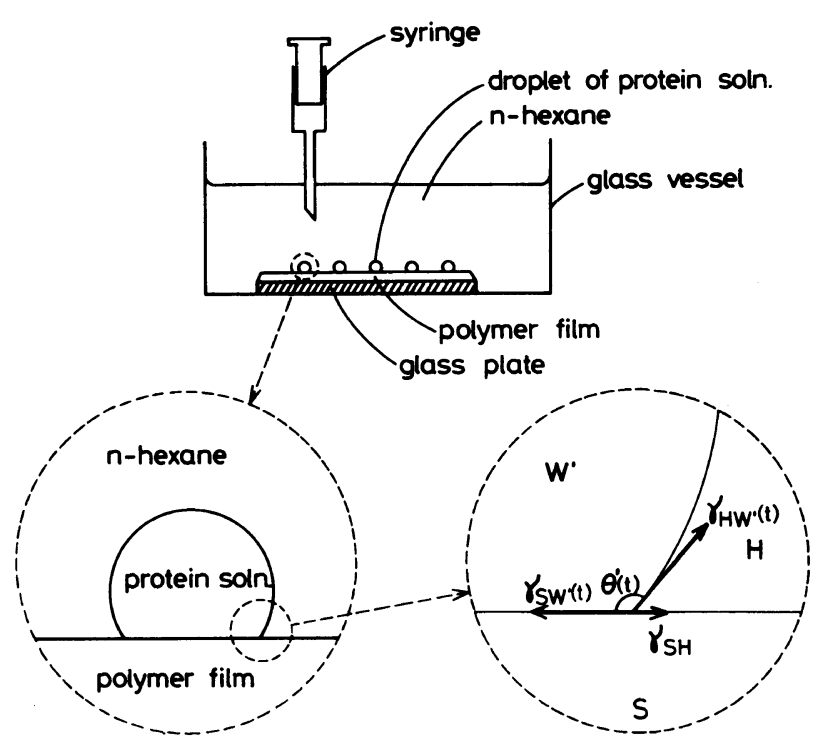

Figure 3. Schematic diagram showing contact angle of protein solution on polymer film in $n$-hexane.

serum $\gamma$-globulin IgG (Cohn fraction II), both purchased from Sigma. These proteins were dissolved in a buffer solution called pseudoextracellular fluid ${ }^{12}$ (PECF) composed of $\mathrm{NaHCO}_{3}, \mathrm{~K}_{2} \mathrm{HPO}_{4}, \mathrm{NaCl}, \mathrm{KCl}$, and water $(I=0.128$ and $\mathrm{pH} 7.4)$.

The polymer samples ${ }^{13,14}$ used were (1) block copolymers composed of $\gamma$-benzyl $\mathrm{L}$ glutamate $(\mathrm{G})$ and L-leucine (L), designated as GLG series, (2) random copolymers composed of $G$ and $L$, designated as GL series, and (3) partly hydrophilic block copolymers, designated as GLG (E) series, derived from GLG films by aminoalcoholysis with 2-amino-1ethanol under the coexistence of octamethylenediamine as a crosslinking agent. Films of GLG and GL polymers were prepared by casting at $25^{\circ} \mathrm{C}$ from chloroform-trifluoroacetic acid $(1 \%)$ solutions.

\section{Measurements}

Bulk compositions of GLG and GL were determined by the elemental analysis, aminoacid analysis, and proton NMR $(90 \mathrm{MHz})$ analysis. The $\mathrm{OH}$ contents of GLG (E) were estimated from IR spectra. The surface com- positions of the polymer films were examined by X-ray photo electron spectroscopy (ESCA), from which the number ratios, $\mathrm{O} / \mathrm{C},=\mathrm{O} / \mathrm{C}$, and $-\mathrm{O}-/ \mathrm{C}$, in the surface layer were estimated.

\section{Method to Determine Interfacial Pressure from Contact Angle}

Figure 3 shows an apparatus to measure contact angles $\theta$ and $\theta^{\prime}$, respectively, of buffer solution and protein solution on polymer film surface in $n$-hexane. A polymer film cast on a glass plate was placed in a glass vessel filled with $n$-hexane. Now a droplet of buffer solution was introduced onto the polymer film via a syringe, and the contact angle $\theta$ was measured as a function of time. In a similar manner, the contact angle $\theta^{\prime}$ of protein solution was measured. Hexane, whose surface tension is $50 \mathrm{dyn}^{-1}$, is a hydrophobic liquid, in which the advancing contact angle of water on polymer surface was confirmed to be in accord with the reciding angle.

As is obvious from Figure 3, the interfacial pressure $\Pi_{\mathrm{sw}}(t)$ of protein solution $\left(w^{\prime}\right)$ on the polymer surface(s) is given by 


$$
\Pi_{\mathrm{sw}^{\prime}}(t)=\gamma_{\mathrm{sw}}-\gamma_{\mathrm{sw}^{\prime}}(t)
$$

where $\gamma_{\mathrm{sw}}$ and $\gamma_{\mathrm{sw}}(t)$ are the interfacial tensions regarding buffer solution $(w)$ and protein solution $\left(w^{\prime}\right)$, respectively, on the solid surface, and the former is confirmed to be independent of time. These two quantities are related to the contact angles by Young-Dupre equation:

$$
\begin{gathered}
\gamma_{\mathrm{sw}^{\prime}}(t)=\gamma_{\mathrm{Hs}}+\gamma_{\mathrm{Hw}^{\prime}}(t) \cos \theta^{\prime}(t) \\
\gamma_{\mathrm{sw}}=\gamma_{\mathrm{Hs}}+\gamma_{\mathrm{Hw}} \cos \theta
\end{gathered}
$$

So, finally we obtain:

$$
\Pi_{\mathrm{sw}^{\prime}}(t)=\gamma_{\mathrm{Hw}} \cos \theta+\gamma_{\mathrm{Hw}^{\prime}}(t) \cos \theta^{\prime}(t)
$$

where subscript $\mathrm{H}$ denotes $n$-hexane. The interfacial tensions $\gamma_{\mathrm{Hw}}$ and $\gamma_{\mathrm{Hw}^{\prime}}(t)$ were measured independently by Wilhelmy Plate method. Thus we determine $\Pi_{\mathrm{sw}}(t)$ by eq 12 from experimentally obtained contact angle and interfacial tension.

\section{RESULTS AND DISCUSSION}

The bulk and surface compositions of GLG and GL samples were shown in Table I together with the contact angle of buffer solution on the polymer surface. Obviously, the contact angle increases with increasing leucine content, hereby the number ratio $-\mathrm{O}-/ \mathrm{C}$ considerably decreases. If we compare the block copolymer GLG with random copolymer GL at the same leucine content $(10.7 \mathrm{~mol} \%)$, the contact angle of GL-34 is considerably higher, and the ratio -O-/C of GL-34 is considerably smaller, than those of GLG-1. Such a finding may lead to that the surface composition of random copolymer is rich in the leucine content than that of block copolymer.

Figure 4 shows the time dependency of interfacial tension $\gamma_{\mathrm{Hw}^{\prime}}$ at $n$-hexane/protein solution interface for BSA of $c_{\mathrm{o}}=0.01,0.025,0.1$, and $0.5 \mathrm{wt} \% \cdot \gamma_{\mathrm{Hw}^{\prime}}$ rapidly decreases at early stage, then slowly approaches to an equilibrium value. For examples, at $c_{\mathrm{o}}=0.1 \mathrm{wt} \%$ and

\begin{tabular}{|c|c|c|c|c|c|}
\hline \multirow{2}{*}{$\begin{array}{l}\text { Sample } \\
\text { code }\end{array}$} & \multirow{2}{*}{$\frac{\text { L-Leu }}{\mathrm{mol}^{\circ} \%}$} & \multirow{2}{*}{$\frac{\text { Contact angle }}{\text { deg }}$} & \multicolumn{3}{|c|}{$\begin{array}{c}\text { ESCA analysis } \\
\text { elemental ratio } \%\end{array}$} \\
\hline & & & $\mathrm{O} / \mathrm{C}$ & $=\mathrm{O} / \mathrm{C}$ & $\mathrm{O}-/ \mathrm{C}$ \\
\hline PBLG $^{a}$ & 0 & $118.8 \pm 0.53$ & 25.0 & 15.6 & 9.4 \\
\hline GLG-1 & 10.7 & $115.4 \pm 0.66$ & 26.0 & 16.4 & 9.7 \\
\hline GLG-2 & 19.1 & $128.1 \pm 0.69$ & 24.1 & 16.5 & 7.6 \\
\hline GLG-3 & 24.9 & $134.5 \pm 0.54$ & 22.9 & 16.1 & 6.8 \\
\hline GLG-4 & 40.0 & $137.3 \pm 0.77$ & 21.6 & 16.1 & 5.5 \\
\hline GL-32 & 5.3 & $123.3 \pm 0.86$ & 23.5 & 15.2 & 8.3 \\
\hline GL-34 & 10.6 & $139.9 \pm 0.89$ & 21.5 & 15.8 & 5.7 \\
\hline GL-38 & 21.0 & $144.5 \pm 0.96$ & 20.9 & 15.4 & 5.5 \\
\hline
\end{tabular}
$t=180 \mathrm{~min}, \gamma_{\mathrm{Hw}^{\prime}}$ is $20.23 \mathrm{dyn} \mathrm{cm}^{-1}$ for $\mathrm{BSA}$,
Table I. Bulk and surface compositions and surface characteristics of PBLG, GLG, and GL

a PBLG, poly( $\gamma$-benzyl L-glutamate).

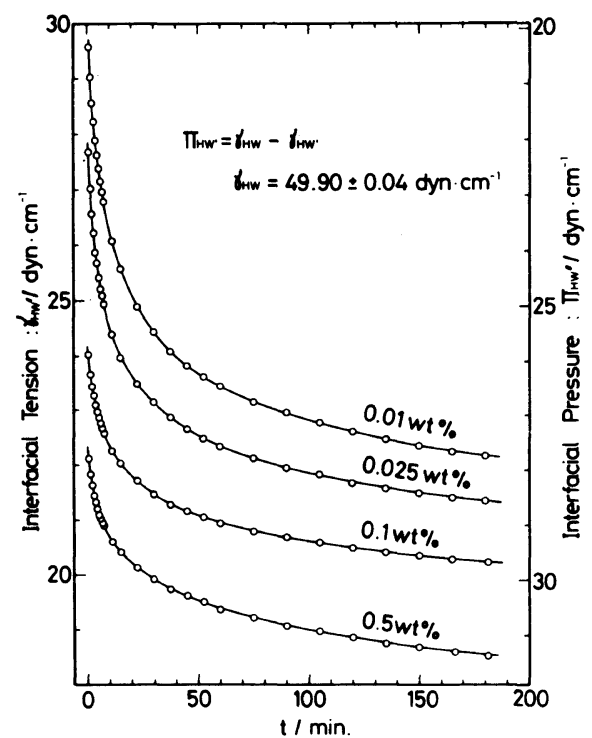

Figure 4. Time dependence of interfacial tension $\gamma_{\mathbf{H w}}$ at $n$-hexane/protein solution interface for BSA of different bulk concentration $c_{\mathrm{o}}$

and $26.5 \mathrm{dyn}^{-1}$ for IgG. But for buffer solution, $\gamma_{\mathbf{H w}}$ is constant independent of time. Figure 5 indicates the contact angles $\theta^{\prime}$ of BSA solution at $c_{\mathrm{o}}=0.1$ and $0.5 \mathrm{wt} \%$, and $\theta$ of buffer solution $\left(c_{0}=0\right)$ on PBLG polymer film as functions of time. Obviously, the contact angle of protein solution rapidly deceases and then slowly approaches to an equilibrium value, but that of buffer solution is constant 


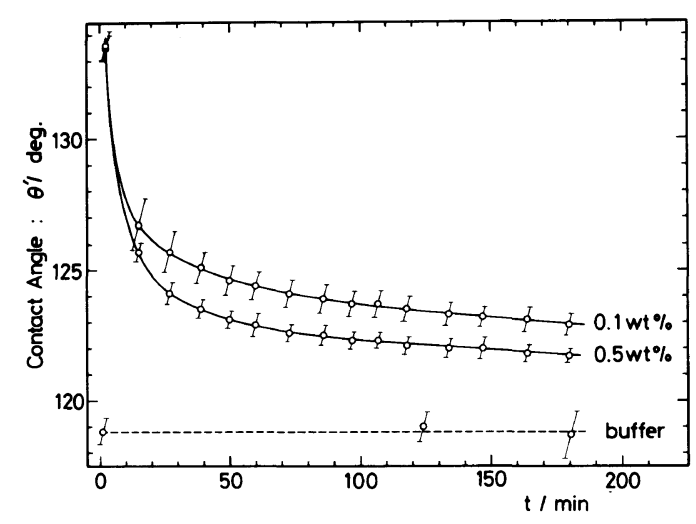

Figure 5. Contact angles of BSA solutions (solid curves) and of PECF (broken curve) plotted against time $t$.

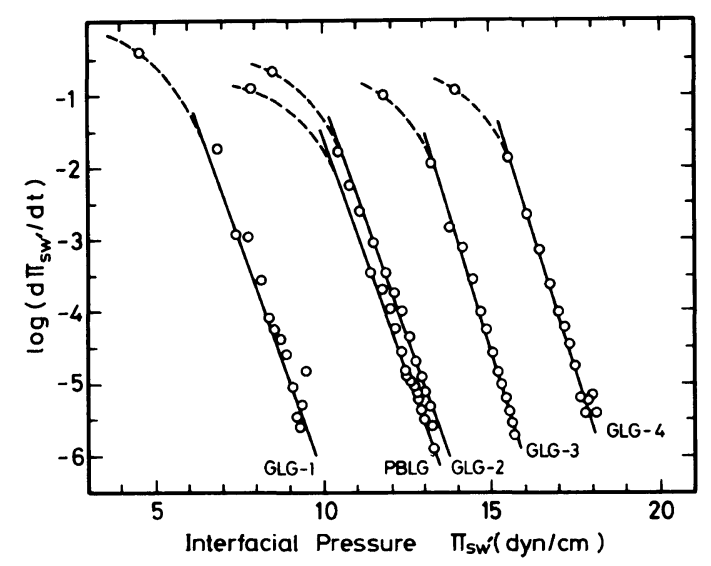

Figure 6. $\log \left(\mathrm{d} \Pi_{\mathrm{sw}} / \mathrm{d} t\right)$ plotted against $\Pi_{\mathrm{sw}^{\prime}}$ for adsorption of $0.1 \mathrm{wt} \%$ BSA solution onto PBLG poly $(\gamma$ benzyl L-glutamate) homopolymer and GLG copolymers.

independent of time. Combining these two experimental results, one obtains $\Pi_{\mathrm{sw}^{\prime}}$ by eq 12 as a function of time.

The interfacial pressures $\Pi_{\mathrm{sw}^{\prime}}$ were plotted against time $t$ for $0.1 \mathrm{wt} \%$ BSA and $\mathrm{IgG}$ solutions. Then the $\Pi_{\text {sw }^{\prime}}$ values were differentiated with time to obtain $\mathrm{d} \Pi_{\mathrm{sw}^{\prime}} / \mathrm{d} t$. In Figures 6 and $7, \log \left(\mathrm{d}_{\mathrm{sw}^{\prime}} / \mathrm{d} t\right)$ values are plotted against $\Pi_{\text {sw' }^{\prime}}$ for GLG-BSA and GLG-IgG systems, respectively. For the adsorption of BSA, in the high interfacial pressure region, a good linear relationship is hold, but in low

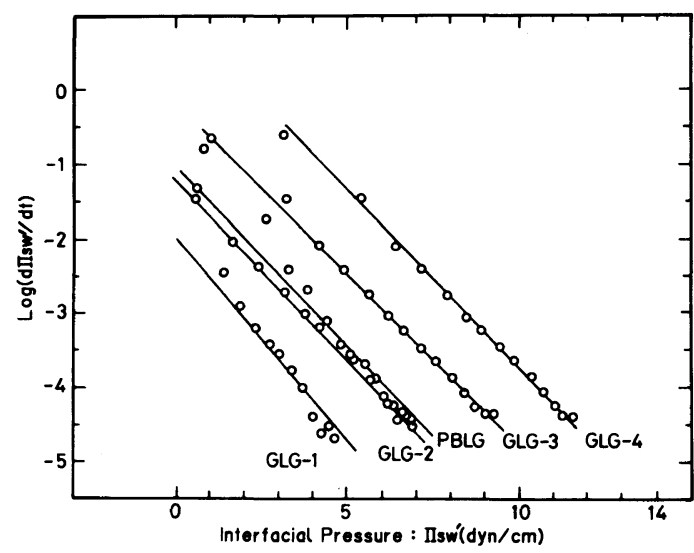

Figure 7. $\log \left(\mathrm{d} \Pi_{\mathrm{sw}} / \mathrm{d} t\right)$ plotted against $\Pi_{\mathrm{sw}^{\prime}}$ for adsorption of $0.1 \mathrm{wt} \%$ IgG solution onto PBLG homopolymer and GLG copolymers.

pressure region, some deviation from the straight line relationship takes place. Such a result means that adsorption behavior at the early stage is diffusion-controlled, but it transposes to an energy barrier-controlled process at the later stage. The straight lines shown in Figure 6 are nearly parallel each other, which means that the adsorption crosssectional $A$ are almost the same.

On the other hand, for the adsorption of $\mathrm{IgG}$, as shown in Figure 7, only straight lines which are parallel each other are obtained. The slopes of the straight lines for IgG are about one third of those for BSA. Though not shown here, we obtained $\log \left(\mathrm{d} \Pi_{\text {sw }^{\prime}}\right)$ $\mathrm{d} t)$ vs. $\Pi_{\mathrm{sw}^{\prime}}$ curves for GL-BSA and GLIgG systems.

Now we briefly refer to the cross-sectional area $A$ occupied in the course of adsorption when a protein molecule approaches the subphase. If an area $A$ necessary for adsorption is occupied, then the adsorption of the molecules takes place. Once. the area is fulfilled, the protein molecules may be adsorbed tightly on the surface by accompanying possible conformational change. In the case of small value of $A$, adsorption is easier to take place in comparison with the case of large value of $A$. In Table III, the interfacial pressure $\Pi_{\text {sw }^{\prime}}$ and the 
area $A$ for PBLG, GLG, and GL systems are shown.

In the next place, we will concern with the hydrophilic block copolymers GLG (E), which were prepared by aminoalcoholysis of GLG-3 in 2-amino-1-ethanol (EA). GLG-3 (2E) and GLG-3 (4E) were obtained by aminoalcoholysis for 2 and 4 hours, respectively. Thus, the $G$ portion of GLG-3 was partly substituted with $\mathrm{OH}$ residues. Table II shows the contact angles of PECF on these films in $n$-hexane. In Figure $8, \log \left(\mathrm{d} \Pi_{\mathrm{sw}^{\prime}} / \mathrm{d} t\right)$ values are plotted against $\Pi_{\mathrm{sw}^{\prime}}$ for the adsorption of $\mathrm{IgG}$ on GLG-3, GLG-3 (2E), and GLG-3 (4E). As is

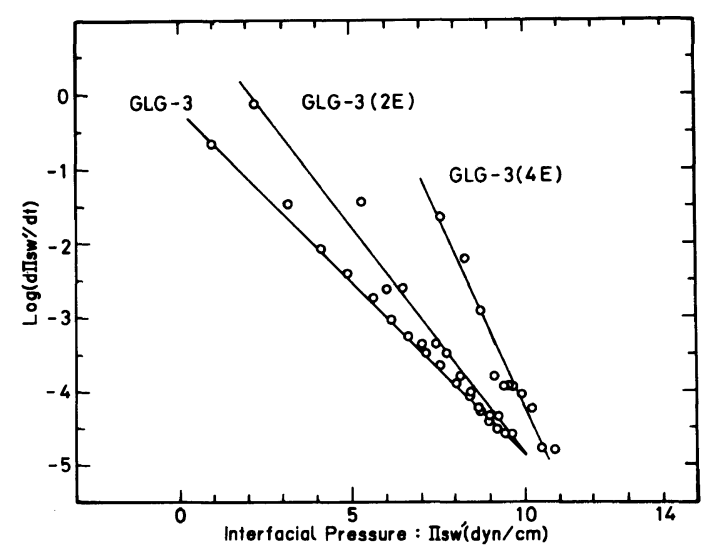

Figure 8. $\log \left(\mathrm{d} \Pi_{\mathrm{sw}} / \mathrm{d} t\right)$ plotted against $\Pi_{\mathrm{sw}^{\prime}}$ for adsorption of $0.1 \mathrm{wt} \%$ IgG solution. onto GLG(E) copolymers of different $\mathrm{OH}$ contents. obvious from the figure, the slopes of straight lines become large with increasing hydrophilicity. This means that $A$ increases with increasing the content of $\mathrm{OH}$ group.

The results of whole experiments are summarized in Table III, in which the $\Pi_{\text {sw }^{\prime}}$ values are those of after 3 hours from the beginning of the experiments. For GLG and GL series, the $A$ values are the same for each protein: $A$ of BSA is $c a$. 5.5, while $A$ of IgG is $c a$. 2. But for GLG (E) series, $A$ values of BSA and of IgG increase with increasing hydrophilicity.

From the experimental results mentioned above, the following conclusions are obtained. The adsorbed amount of protein by diffusioncontrolled process was larger with BSA than with IgG for both GLG and GL series. The $\Pi_{\mathrm{sw}^{\prime}}$ values at 3 hours of BSA and $\mathrm{IgG}$ for random copolymers were higher than the respective values for block copolymers of the same leucine content. This means that GL

Table II. GLG (E) samples carrying hydrophilic groups

\begin{tabular}{|c|c|c|c|}
\hline \multirow{2}{*}{ Sample code } & L-Leu & $\gamma$-BLG & Contact angle \\
\hline & $\mathrm{mol}^{\circ} \%$ & $\mathrm{~mol}^{\circ} \%$ & $\operatorname{deg}$ \\
\hline GLG-3 & 24.9 & 75.1 & $134.5 \pm 0.54$ \\
\hline GLG-3 (2E) & 24.9 & ( $2 \mathrm{~h}$ in $\mathrm{EA})$ & $120.3 \pm 1.86$ \\
\hline GLG-3 (4E) & 24.9 & ( $4 \mathrm{~h}$ in $\mathrm{EA})$ & $112.2 \pm 1.06$ \\
\hline
\end{tabular}

Table III. Surface pressure $\Pi_{\mathrm{sw}^{\prime}}$ and surface area $A$ of BSA and IgG on PBLG, GLG, GL, and GLG(E) surfaces

\begin{tabular}{|c|c|c|c|c|c|c|}
\hline \multirow{2}{*}{$\begin{array}{l}\text { Sample } \\
\text { code }\end{array}$} & \multirow{2}{*}{$\frac{\text { L-Leu }}{\mathrm{mol}^{\circ} \%}$} & \multirow{2}{*}{$\frac{\gamma-\mathrm{BLG}}{\mathrm{mol} \%}$} & \multicolumn{2}{|c|}{$\Pi_{\mathrm{sw}^{\prime}} / \mathrm{dyn} \mathrm{cm}^{-1}$} & \multicolumn{2}{|c|}{$A / \mathrm{nm}^{2}$} \\
\hline & & & BSA & $\mathrm{IgG}$ & BSA & IgG \\
\hline PBLG & - & 100.0 & $13.1 \pm 0.12$ & $7.1 \pm 0.23$ & 6.0 & 2.0 \\
\hline GLG-1 & 10.7 & 89.3 & $9.5 \pm 0.20$ & $4.8 \pm 0.18$ & 5.6 & 2.1 \\
\hline GLG-2 & 19.1 & 80.9 & $13.3 \pm 0.10$ & $6.9 \pm 0.10$ & 5.5 & 1.9 \\
\hline GLG-3 & 24.9 & 75.1 & $15.7 \pm 0.14$ & $9.3 \pm 0.04$ & 6.2 & 1.9 \\
\hline GLG-4 & 40.0 & 60.0 & $18.1 \pm 0.05$ & $11.6 \pm 0.12$ & 5.8 & 1.9 \\
\hline GL-32 & 5.3 & 94.7 & $12.4 \pm 0.13$ & $5.2 \pm 0.07$ & 5.4 & 2.1 \\
\hline GL-34 & 10.6 & 89.4 & $19.3 \pm 0.05$ & $12.7 \pm 0.08$ & 5.6 & 1.9 \\
\hline GL-38 & 21.0 & 79.0 & $21.7 \pm 0.06$ & $15.2 \pm 0.06$ & 6.0 & 1.9 \\
\hline GLG-3 (2E) & 24.9 & & $9.9 \pm 0.63$ & $9.7 \pm 0.51$ & 6.5 & 2.3 \\
\hline GLG-3 (4E) & 24.9 & & $4.4 \pm 0.42$ & $10.9 \pm 0.48$ & 8.0 & 4.7 \\
\hline
\end{tabular}


surface is more hydrophobic than GLG surface of the same bulk composition. The effective cross-sectional area $A$ of BSA $\left(c a .6 .0 \mathrm{~nm}^{2}\right)$ for both GLG and GL series was about three times of $A$ of $\operatorname{IgG}\left(c a .2 .0 \mathrm{~nm}^{2}\right.$ ), and $\Pi A$ for BSA was always higher than that for IgG. This means that IgG is more easily adsorbed in the energy barrier-controlled process. For GLG (E) which partly carrying hydrophilic groups, the adsorbed amount of IgG in the early stage is larger than that of BSA, and $A$ value of $\operatorname{IgG}$ $\left(4.7 \mathrm{~nm}^{2}\right)$ was larger than that of parent GLG$3\left(1.9 \mathrm{~nm}^{2}\right)$. From these findings, we suggest that the $F_{a b}$ portion of $\gamma$-globulin orients toward the hydrophilic surface by end-on fashion, in contrast to the orientation of $F_{c}$ portion by end-on fashion toward the hydrophobic surface.

Acknowledgment. The authors wish to thank Mr. T. Makino for his cooperation in the experiments.

\section{REFERENCES}

1. A. F. H. Ward and L. Tordai, J. Chem. Phys., 14, 453 (1946).

2. A. F. H. Ward and L. Tordai, Recueil, 71, 572 (1952).

3. F. MacRitchie and A. E. Alexander, J. Colloid Sci., 18, 458 (1963).

4. E. Tornberg, J. Colloid Interface Sci., 64, 391 (1978).

5. D. E. Graham and M. C. Phillips, J. Colloid Interface Sci., 70, 403 (1979).

6. A. J. I. Ward and L. H. Regan, J. Colloid Interface Sci., 78, 389 (1980).

7. I. F. Miller, J. Colloid Interface Sci., 86, 539 (1982).

8. R. S. Hansen, J. Colliod Sci., 16, 549 (1961).

9. J. F. Baret, J. Chem. Phys., 65, 895 (1968).

10. J. G. Petrov and R. Miller, Colloid Polym. Sci., 255, 669 (1977).

11. P. Dulm and W. Nord, J. Colloid Interface Sci., 91, 248 (1983).

12. C. A. Homsey, J. Biomed. Mater. Res., 4, 341 (1970).

13. A. Nakajima, T. Hayashi, K. Kugo, and K. Shinoda, Macromolecules, 12, 840 (1979).

14. T. Hayashi, Y. Tabata, K. Takeshima, and A. Nakajima, Polym. J., 17, 1149 (1985). 